\title{
False increased vitamin B12 levels in autoimmune gastritis and subacute combined degeneration of the spinal cord
}

\author{
Cătălina Elena Bistriceanu*, Florentina Anca Danciu, Lucian Crăcană \\ Neurology Department, "Prof. Dr. N.Oblu” Neurosurgery Clinical Emergency Hospital, lasi, Romania
}

\begin{abstract}
Due to the fact that vitamin B12 is important in the myelination of the central nervous system, vitamin B12 deficiency is associated with neurologic and psychiatric manifestations. It causes reversible megaloblastic anemia and the most frequent cause of severe vitamin B12 deficiency is the loss of the intrinsic factor in autoimmune atrophic gastritis. Vitamin B12 deficiency can cause both the demyelination of the cervical, dorsal and lateral columns of the spinal cord, and the demyelination of white matter in the brain. We report a case of a 34 year-old man who was admitted in our clinic with ataxic paraparesis and schizoaffective structure with depressive decompensations. Laboratory exams detected a severe hyporegenerative macrocytic anemia and increased values of vitamin B12 (> $2000 \mathrm{pg} / \mathrm{mL})$. Clinical and paraclinical tests that showed improvement under B12 treatment became paradoxical and raised many questions. We found in the literature that it is possible that a false normal vitamin B12 level may have been caused by interference from a high-titer of intrinsic factor antibody. The patient was diagnosed with autoimmune gastritis and secondary loss of intrinsic factor in the gastroenterology department and he had a complete remission of the symptoms and the hematological parameters after vitamin B12 treatment.
\end{abstract}

Keywords: vitamin B12 deficiency, megaloblastic anemia, subacute combined degeneration, autoimmune gastritis, intrinsic factor antibody

\section{Introduction}

Due to the fact that vitamin B12 is important in the myelination of the central nervous system, vitamin B12 deficiency is associated with neurologic and psychiatric manifestations. It causes reversible megaloblastic anemia and the most frequent cause of severe vitamin B12 deficiency is the loss of the intrinsic factor in autoimmune atrophic gastritis.

Received: February 2015; Accepted after review: March 2015; Published: March 2015.

*Corresponding author: Catalina Elena Bistriceanu, MD, Neurology Department, "Prof. Dr. N. Oblu" Neurosurgery Clinical Emergency Hospital, No 2 Ateneului st., lasi, Romania.

Email: catalina nastac@yahoo.com
Vitamin B12 deficiency can cause both the demyelination of the cervical, dorsal and lateral columns of the spinal cord, and the demyelination of white matter in the brain [1, 2]. The most common hematologic finding in B12 deficiency is the megaloblastic anemia with an elevated mean corpuscular volume and mean corpuscular hemoglobin. The peripheral blood smear can show oval macrocytes, immature megaloblastic nucleated red cells and hypersegmented neutrophils.

Considering that in cases of severe pancytopenia with increased erythroblasts and cytogenetic abnormalities, using bone marrow biopsy and aspiration may be misleading for megaloblastic anemia because the diagnosis can be confused with acute leukemia [3, 4].

Frequent neurologic manifestations associated with B12 deficiency are spinal cord manifestations (subacute combined 
degeneration of the spinal cord, ataxia, spasticity and abnormal gait), peripheral neuropathies, optic neuropathy and cerebrovascular diseases. Psychiatric symptoms may be confusion, memory changes, depression, dementia, acute psychosis, delirium with/without hallucinations and/or delusions, reversible maniac and schizophreniform states (megaloblastic madness). The loss of intrinsic factor caused by autoimmune atrophic gastritis ("pernicious anemia") is one of the most frequent causes of severe vitamin B12 deficiency $[5,6]$. It is an autoimmune gastritis associated with lack of intrinsic factor due to the destruction of the parietal cells from the stomach.

\section{Case report}

We report a case of a 34 year-old man with antecedents of meningitis in childhood, without any treatment at home, who came into our clinic for unsteady gait, difficulty in walking, distal motor deficit, numbness and paresthesia of his lower limbs. The symptomatology started one month before admission and had an ascending evolution.

$\mathrm{He}$ was first admitted into the infectious disease department because of the worsened motor symptoms during a fever episode. At that time, the neurosurgical exam and the spinal MRI (lumbar) were clear. The suspicion of sepsis was ruled out by the infectious disease department and a severe hyporegenerative macrocytic anemia was discovered. Because the neurological status of the patient worsened, he was transferred into neurology clinic to continue the investigations.

Clinical examination on admittance revealed: pale yellow waxy skin, red and smooth appearance of the tongue (glossitis); no cardiopulmonary modifications.

The neurologic examination revealed: flaccid paraparesis (symmetrical distal motor deficit of the lower limbs with $M R C=4 / 5$ bilaterally; muscle hypotonia with unsteady gait, possible with support and loss of reflexes; ataxia of the lower limbs; bilaterally Babinski sign); hypoesthesia with the level of sensitivity at D6; loss of vibratory sensitivity and proprioception; the abolition of the middle and inferior abdominal reflex. He had no sphincter disorder and no abnormality of cranial nerves (Table 1).

The psychological examination revealed a schizophrenic state with depressive decompensations and sensory dissociative elements with phobic and obsessive aspect; mild memory loss and attention difficulties.

We were facing a progressive paraparesis with the level of sensitivity at D6 and a severe hyporegenerative macrocytic anemia. The next step was to determine the level of vitamin B12 and folic acid. After this, we started the treatment with vitamin B12 and folic acid.

In the meantime, we continued the investigations to exclude other disorders. A CT scan was performed to exclude a parasagittal meningioma or a bilateral lesion in anterior cerebral artery territory.

Some laboratory exams were added, such as peripheral blood smears which showed macrocytic erythrocytes, platelets with normal aspect and rare reactive lymphocytes.

The leukocyte formula showed segmented neutrophils 49\%; eosinophils 2\%; basophils $1 \%$; lymphocytes $38 \%$; monocytes $10 \%$. Blood analysis showed an increased value of $\mathrm{LDH}$ $4172 \mathrm{U} / \mathrm{L}$ (normal values are 135-225 U/L), a severe hyporegenerative macrocytic anemia with hemoglobin of $7.7 \mathrm{~g} / \mathrm{dL}$ and low values of erythrocytes $(2.210 .000 / \mu \mathrm{L})$ and leukocytes $(3.200 .000 / \mu \mathrm{L})$. We considered the possibility of a myelodysplastic syndrome, but the patient refused a medullary puncture. Few days later, when other results arrived, the protein electrophoresis was normal.

A spinal MRI (dorsal) was performed to exclude a progressive vascular, infectious, tumoral or traumatic lesion of the spinal cord. A mild reduction with $5 \mathrm{~mm}$ of the anteroposterior medullary diameter from D5 to D7 was found (Figure 1). 
Table 1. Vitamin B12 deficiency: neurological, psychiatric, hematologic disorders [7]

\begin{tabular}{|c|c|}
\hline Spinal cord & $\begin{array}{l}\text { Myelopathy } \\
\text { Spongy degeneration } \\
\text { Paresthesias } \\
\text { Loss of vibration, position, ataxic gait, limb weakness; spasticity (hyperreflexia); } \\
\text { positive Romberg sign; Lhermitte's sign; segmental cutaneous sensory level }\end{array}$ \\
\hline Autonomic nervous system & $\begin{array}{l}\text { Postural hypotension } \\
\text { Incontinence } \\
\text { Impotence }\end{array}$ \\
\hline Peripheral nervous system & $\begin{array}{l}\text { Cutaneous sensory loss } \\
\text { Hyporeflexia } \\
\text { Symmetric weakness } \\
\text { Paresthesias }\end{array}$ \\
\hline Psychiatric disorders & $\begin{array}{l}\text { Altered mental status } \\
\text { Cognitive defects } \\
\text { "Megaloblastic madness": depression, mania, irritability, paranoia, delusions, } \\
\text { lability }\end{array}$ \\
\hline Peripheral blood & $\begin{array}{l}\text { Macrocytic red cells, macroovalocytes } \\
\text { Anisocytosis, fragmented forms } \\
\text { Hypersegmented neutrophils, } \\
\text { Leukopenia, possible immature white cells } \\
\text { Thrombocytopenia, Pancytopenia } \\
\text { Elevated lactate dehydrogenase level (extremes possible) } \\
\text { Elevated indirect bilirubin and aspartate aminotransferase levels } \\
\text { Decreased haptoglobin level } \\
\text { Elevated levels of methylmalonic acid, homocysteine, or both }\end{array}$ \\
\hline Bone Marrow & $\begin{array}{l}\text { Hypercellular, increased erythroid precursors } \\
\text { Open, immature nuclear chromatin } \\
\text { Dyssynchrony between maturation of cytoplasm and nuclei } \\
\text { Giant bands, metamyelocytes } \\
\text { Karyorrhexis, dysplasia } \\
\text { Abnormal results on flow cytometry and cytogenetic analysis }\end{array}$ \\
\hline Other sensorial deficits & Optic atrophy, anosmia, loss of taste, glossitis \\
\hline
\end{tabular}
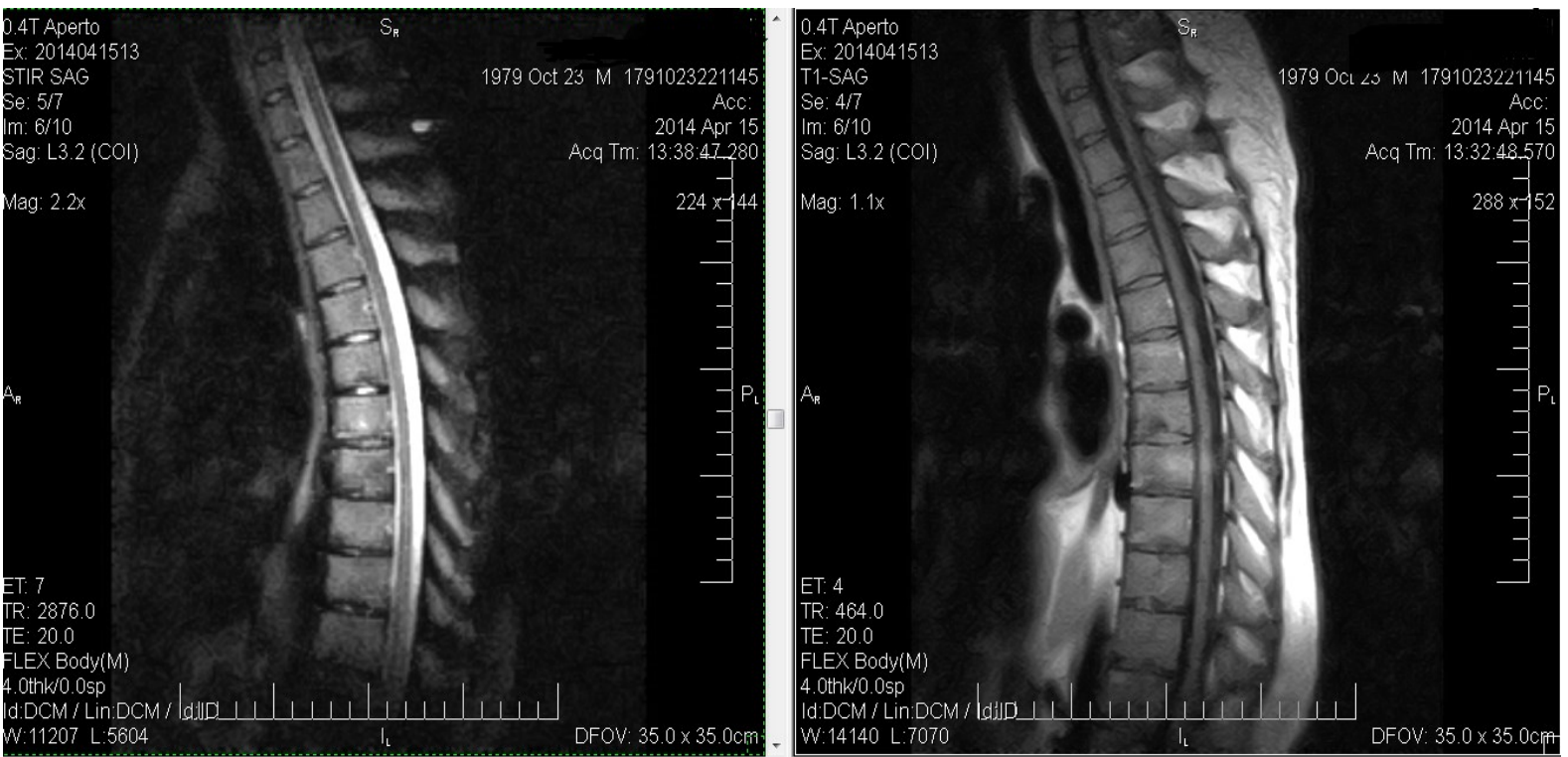

Fig. 1. Spinal MRI (dorsal - T2 weighted sagittal): the reduction with $5 \mathrm{~mm}$ of the anteroposterior medullary diameter from D5 to D7 
Unfortunately, the patient refused a lumbar puncture with a CSF exam, so a Miller Fisher subacute polyradiculoneuritis could not be directly excluded. Despite his refusal, negative Lasegue and Bonnet, the absence of sphincter disorder and the absence of inflammation of spinal roots on MRI made this diagnosis less probable. We considered that the clinical and paraclinical tests aspects were highly suggestive for subacute combined degeneration of the spinal cord and we continued the treatment with B12 vitamin and folic acid. Under this treatment we obtained an improvement of the general and neurological state of the patient. The results of the B12 level and folic acid (prior to the administration of the treatment) were: Vitamin B12 - 9678 $\mathrm{pg} / \mathrm{ml}$ (normal value: 191-663pg/ml); Folate $43,8 \mathrm{ng} / \mathrm{ml}$ (normal value: $4,6-18,7 \mathrm{ng} / \mathrm{ml}$ ). The analysis was repeated in another laboratory and we obtained a level of B12 vitamin $>2000 \mathrm{pg} / \mathrm{mL}$. High levels of B12 could also be explained, to some extent, by either one of innate errors (hereditary enzymatic disorders), autoimmune atrophic gastritis, neoplastic disorders of the digestive tract or some myelodysplastic syndromes. The clinical and paraclinical test improvements under treatment helped us decide to continue the same therapy under careful observation. Therefore, we obtained the following values in almost 2 weeks of treatment (Table 2).

Table 2. Initial hematologic values and after 2 weeks of vitamin B12 treatment

\begin{tabular}{lll}
\hline & Initial & After \\
\hline Hemoglobin & $7.8 \mathrm{~g} / \mathrm{dl}$ & $10.8 \mathrm{~g} / \mathrm{dl}$ \\
$\mathrm{LDH}$ & $4172 \mathrm{U} / \mathrm{L}$ & $629 \mathrm{U} / \mathrm{L}$ \\
MCV & $102 \mathrm{fL}$ & $95.1 \mathrm{fL}$ \\
Leucocytes & $3.200 .000 / \mu \mathrm{L}$ & $4.600 .000 / \mu \mathrm{L}$ \\
Erythrocytes & $2.210 .000 / \mu \mathrm{L}$ & $3.490 .000 / \mu \mathrm{L}$ \\
\hline
\end{tabular}

After 2 weeks, the psychiatric symptoms, the motor deficit and paresthesias of the lower limbs decreased in intensity; therefore the patient was able to walk without support.

After being discharged, the patient had a gastroenterological exam with endoscopy and the biopsy of the gastric mucosa. Intestinal metaplasia into the gastric body was observed. He was diagnosed with atrophic autoimmune gastritis and secondary loss of intrinsic factor and he was recommended to continue the B12 vitamin treatment.

Two months later, upon readmission into the neurology clinic, the patient didn't have any motor deficit and psychiatric symptoms. $\mathrm{He}$ had no abnormalities in the laboratory exam: $\mathrm{Hb}=13.6 \mathrm{~g} / \mathrm{dl} ; \mathrm{MCV}=79.1 \mathrm{fL}$; leucocytes, erythrocytes - normal values.

\section{Discussions}

It is possible that a false normal vitamin B12 level may have been caused by interference from a high-titer of intrinsic factor antibody [8].

In our case, the highly suggestive clinical aspect of B12 deficiency and the marked macrocytic anemia was a compelling proof of a deficit of B12 with neurologic, psychiatric and hematologic manifestations. The peripheral blood smear showed macrocytic erythrocytes.

We consider that the increased values of B12 vitamin were due to a high titer of intrinsic factor antibody. The arguments are the clinical and laboratory aspects compatible with pernicious anemia and the complete remission of the symptomatology under cobalamin treatment.

The main problem with chemically removing the anti-intrinsic factor antibodies (common in pernicious anemia) in automated assays is that the intrinsic factor is used as the assay binding protein. Recent studies show normal values or falsely high values of vitamin B12 in many patients with pernicious anemia $[2,9]$. 
If cobalamin levels are uncertain, the measurement of homocysteine levels and serum methylmalonic acid (accumulated in B12 deficiency) can be done when having a high clinical suspicion $[10,11]$. The usefulness of the above mentioned metabolites could be limited in renal insufficiency when elevated. Only elevated homocysteine levels might be the cause for lack of folate or vitamin B6 [1113].

\section{References}

1. Healton EB, Savage DG, Brust JC, Garrett TJ, Lindenbaum J. Neurologic aspects of cobalamin deficiency. Medicine 1991; 70:229245.

2. R, Agrawal YP. Failures of cobalamin assays in pernicious anemia. N Engl J Med 2012; 367:385-386.

3. Andrès $\mathrm{E}$, Affenberger $\mathrm{S}$, Zimmer $\mathrm{J}$, et al. Current hematological findings in cobalamin deficiency: a study of 201 consecutive patients with documented cobalamin deficiency. Clin Lab Haematol 2006; 28:50-56.

4. Parmentier $\mathrm{S}$, Meinel $\mathrm{J}$, Oelschlaegel $U$, et al. Severe pernicious anemia with distinct cytogenetic and flow cytometric aberrations mimicking myelodysplastic syndrome. Ann Hematol 2012; 91:1979-1981.

5. Toh BH, Chan J, Kyaw T, Alderuccio F. Cutting edge issues in autoimmune gastritis. Clin Rev Allergy Immunol 2012; 42:269-278.

6. Lindenbaum J, Healton EB, Savage DG, et al. Neuropsychiatric disorders caused by cobalamin deficiency in the absence of anemia or macrocytosis. N Engl J Med 1988; 318:1720-1728.

7. Sally $\mathrm{P}$, Stabler MD. Vitamin B12 Deficiency. $N$ Engl J Med 2013; 368:149-160.

\section{Conclusions}

In general, rapid diagnosis and supplementation in vitamin B12 deficiency may prevent permanent complications. This implies that it must be considered for patients with hematologic disorders and/or progressive neuropsychiatric signs [14].

We consider that the presented case represents an argument for the importance of clinical sense and the limitations of laboratory methods.

8. Scarpa E, Candiotto L, Sartori R, et al. Undetected vitamin B12 deficiency due to false normal assay results. Blood Transfus 2013; 11:627-629.

9. Yang DT, Cook RJ. Spurious elevations of vitamin B12 with pernicious anemia. N Engl J Med 2012; 366:1742-1743.

10. Oh R, Brown DL. Vitamin B12 deficiency. Am Fam Physician 2003; 67:979-986.

11. Hvas AM, Nexo E. Diagnosis and treatment of vitamin B12 deficiency-an update. Haematologica 2006; 91:1506-1512.

12. Solomon LR. Cobalamin-responsive disorders in the ambulatory care setting: unreliability of cobalamin, methylmalonic acid, and homocysteine testing. Blood 2005; 105:978985.

13. Hvas AM, Juul S, Gerdes LU, Nexø E. The marker of cobalamin deficiency, plasma methylmalonic acid, correlates to plasma creatinine. J Intern Med 2000; 247:507-512.

14. Nataliya Mar, David Askin, Robert J. Klafter. Pitfalls in the diagnosis of Vitamin B12 deficiency. Open Journal of Hematology, Volume 3, 2012. 